\title{
Editorial
}

\section{Patient Safety vis-à-vis Safe Surgery: The Road Less traveled}

In October 2004, the World Health Organization (WHO) launched the World Alliance for Patient Safety in response to World Health Assembly Resolution 55.18 urging WHO and member states to pay the closest possible attention to the problem of patient safety. The Alliance raised awareness and political commitment to improve the safety of care and supports member states to develop patient safety policy and practice.

The global patient safety challenge aims at bringing together the expertise of specialists to improve the safety of care. The area chosen, for the first challenge in 2005 to 2006, was infection associated with healthcare (HAI). The problem area selected for the second global patient safety challenge, in 2007 to 2008, was the Safety of Surgical Care.

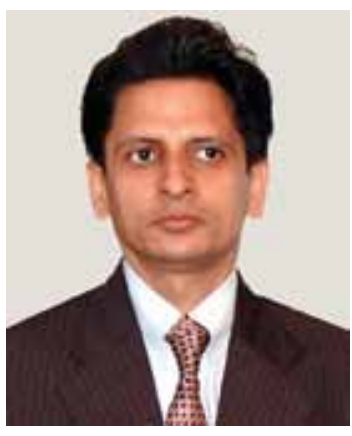

Surgical care has been an essential component of healthcare worldwide since long. As the incidences of traumatic injuries, cancers and cardiovascular disease continue to rise, the impact of surgical intervention on public health systems will only continue to swell. Surgery is often one of the significant therapy that can alleviate disabilities and reduce the risk of death from common conditions. Every year, millions of people undergo surgical treatment, and surgical interventions account for an estimated $13 \%$ of the world's total disability-adjusted life years (DALYs). ${ }^{1}$ While surgical procedures are intended to save lives, unsafe surgical care can result into substantial harm. Given the ubiquity of surgery, this has significant implications for public health. ${ }^{2}$

Five facts about surgical safety are as follows:

- Complications after inpatient operations occur in up to $25 \%$ of patients.

- The reported crude mortality rate after major surgery is 0.5 to $5 \%$.

- In industrialized countries, nearly half of all adverse events in hospitalized patients are related to surgical care.

- At least half of the cases in which surgery led to harm are considered to be preventable.

- Known principles of surgical safety are inconsistently applied even in the most sophisticated settings.

An estimated 234 million major operations are performed around the world each year, corresponding to one operation for every 25 people alive. ${ }^{3}$ Each year, an estimated 63 million people undergo surgical treatment due to traumatic injuries, another 10 million operations are performed for pregnancy-related complications, and 31 million more are undertaken to treat malignancies. ${ }^{4}$ In industrialized countries, studies suggest that major complications are reported to occur in 3 to $16 \%$ of inpatient surgical procedures, with permanent disability or death rates of approximately 0.4 to $0.8 \%$. In developing countries, studies suggest a death rate of 5 to $10 \%$ during major surgery. ${ }^{5}$

Ipso facto, interpolating the same to absolute numbers, minimum seven million surgical patients could be harmed by surgical complications each year including at least one million patients who could die during or immediately following a procedure. Not only this, mortality from general anesthesia alone is reported to be as high as one in 150 in some parts of sub-Saharan Africa. ${ }^{6}$ In developed countries, studies confirm the magnitude and pervasiveness of the problem. In the developing world, the poor state of infrastructure and equipment, unreliable supplies and quality of medications, shortcomings in organizational management and infection control, inadequate capacity and training of personnel and severe underfinancing, all contribute to this disdain.

The working group on problem area selected (safety of surgical care) for the second global patient safety challenge, in 2007 to 2008, reached consensus on four areas in which dramatic improvements could be achieved, as regards safety of surgical care. These are the following:

- Surgical site infection prevention

- Safe anesthesia

- Safe surgical teams and

- Measurement of surgical services

\section{Surgical Site Infection Prevention}

Surgical site infections remain one of the most common causes of serious surgical complications. Evidence shows that proven measures, such as antibiotic prophylaxis within hour before incision and effective sterilization of instruments, are inconsistently resorted to. This is often not because of the cost or lack of resources but because of lack of system in place. For example, antibiotics are given perioperatively in both the developed and developing countries 
but they are often administered too early, too late or simply erratically, making them less effective in mitigating patient harm.

\section{Safe Anesthesia}

Anesthetic complications remain a substantial cause of surgical death globally, despite safety and monitoring standards which have significantly reduced unnecessary deaths and disability in developed countries. Three decades ago, a patient undergoing general anesthesia had an estimate done in 5000 chance of death. With improvements in knowledge and basic standards of care, the risk has dropped to one in 200,000 in the developed worlda 40-fold improvement. Unfortunately, the rate of anesthesia-associated mortality in developing countries appears to be 100 to 1000 times higher, indicating a serious, sustained lack of safe anesthesia for surgery in these settings. Published series showing avoidable anesthesia mortality rates of 1:3000 in Zimbabwe, ${ }^{7} 1: 1900$ in Zambia, ${ }^{8} 1: 500$ in Malawi $^{9}$ and 1:150 in Togo, ${ }^{10}$ demonstrate a serious, sustained absence of safe anesthesia for surgery.

\section{Safe Surgical Teams}

Teamwork is the fundamental tenet of all effectively functioning systems involving multiple people. In the operating room, where tension may be high and lives are at stake, teamwork is an essential component of safe surgery. The quality of teamwork depends on the culture of the team and its communication patterns as well as the clinical skills and situational sensitivity of the team members. Improving team characteristics should aid communication and reduce patient harm.

\section{Measurement of Surgical Services}

A major problem in surgical safety has been a shortage of basic data. Efforts to reduce maternal and neonatal mortality during childbirth have been critically reliant on routine surveillance of mortality rates and systems of obstetric care to monitor successes and failures. Similar surveillance has not been undertaken for surgical care as is needed. Data on surgical volume are available for only few countries and are not standardized. Routine surveillance to evaluate and measure surgical services must be established if public health systems are to ensure progress in improving the safety of surgical care.

Further, the working group on problem area selected (safety of surgical care) for the second global patient safety challenge, in 2007 to 2008, also reached consensus in defining ten essential objectives that should be met by every surgical team during surgical care. These include: the team will operate on the correct patient at the correct site, use methods known to prevent harm from anesthetic administration, while protecting the patient from pain, recognize and effectively prepare for life-threatening loss of airway or respiratory function, recognize and effectively prepare for risk of high blood loss, avoid inducing an allergic or adverse drug reaction known to be a significant risk to the patient, consistently use methods known to minimize risk of surgical site infection, prevent inadvertent retention of sponges or instruments in surgical wounds, secure and accurately identify all surgical specimens, effectively communicate and exchange critical patient information for the safe conduct of the operation and hospitals and public health systems will establish routine surveillance of surgical capacity, volume and results.

In industrialized countries, the rate of major complications has been documented to occur in 3 to $22 \%$ of inpatient surgical procedures, and the death rate 0.4 to $0.8 \%{ }^{11,12}$ Nearly half the adverse events in these studies were determined to be preventable. Studies in developing countries suggest a death rate of 5 to $10 \%$ associated with major surgery. ${ }^{13,14}$ Infections and other postoperative complications are also a serious concern around the world.

Avoidable surgical complications thus account for a large proportion of preventable medical injuries and deaths globally. Adverse events have been estimated to affect 3 to $16 \%$ of all hospitalized patients, and more than half of such events are known to be preventable. ${ }^{15-18}$ Despite dramatic improvements in surgical safety knowledge, at least half of the events occur during surgical care. ${ }^{19}$ Assuming a 3\% perioperative adverse event rate and a $0.5 \%$ mortality rate globally, almost seven million surgical patients suffer significant complications each year. Surgical safety has, therefore, emerged as a significant global public health concern. Just as public health interventions and educational projects have dramatically improved maternal and neonatal survival, analogous efforts might improve surgical safety and quality of care.

There are few underlying challenges to improving surgical safety. First, it has not yet been recognized as a significant public health concern. Because of often high expense of surgical care, it is assumed to be of limited relevance in poor- and middle-income countries; however, the WHO global burden of disease report in 2002 
showed that a significant proportion of the disability from disease in the world is due to conditions that are treatable by surgical intervention. Debas et al estimated that $11 \%$ of the 1.5 billion disability-adjusted life years are due to diseases treatable by surgery. Problems associated with surgical safety are well recognized in developed and rather test in developing countries.

The second underlying problem in improving surgical safety has been paucity of adequate and reliable data. Efforts to reduce maternal and neonatal mortality at childbirth have relied critically on routine surveillance of mortality rates and systems of obstetric care, so that successes and failures could be monitored and recognized. Similar surveillance has been widely lacking for surgical care. The WHO Patient Safety Program found that data on surgical volume were available for only a minority of WHO member states.

The third underlying problem in ensuring surgical safety is that existing safety practices do not appear to be used reliably in any country. Lack of resources is an issue in low-income countries, but it is not necessarily the most important factor. For example, surgical site infection remains one of the most common causes of serious surgical complications, yet evidence indicates that proven measures, such as antibiotic prophylaxis immediately before incision and confirmation of effective sterilization of instruments, are inconsistently followed. This is not because of cost but because of lack of systems in place.

Last but not the least, measurement of impact is a key component of this challenge. Meaningful metrics ought to be identified, even if they relate only to surrogate processes, and they must be reasonable and quantifiable by stakeholders in every way. If the principles of simplicity, applicability and measurability are followed, the goal of successful implementation can be achieved easily.

There is no single remedy available which can improve surgical safety. It calls for reliable completion of a sequence of necessary steps in care, not just by the surgeons but by the team of healthcare professionals working in sync within a supportive healthcare delivery system for the benefit of the patient, which is the end objective.

\section{REFERENCES}

1. WHO_Patient safety/safe surgery. Available at: http://www.who.int/patientsafety/safesurgery/en/. Accessed on: 08-03-15 at $1300 \mathrm{hrs}$.

2. WHO—Patient safety/safe surgery. Available at: http://www.who.int/patientsafety/safesurgery/en/. Accessed on: 08-03-15 at $1300 \mathrm{hrs}$.

3. Safe surgery save lives-WHO/IER/PSP/2008.07. World Health Organization; 2008. Reprint 2009.

4. Debas H, et al. Surgery. In: Disease control priorities in developing countries. 2nd ed. Washington DC, International Bank for Reconstruction and Development and The World Bank Disease Control Priorities Project; 2006.

5. Safe surgery save lives-WHO/IER/PSP/2008.07, World Health Organization; 2008. Reprint 2009.

6. Ouro-Bang'na Maman AF, et al. Deaths associated with anaesthesia in Togo, West Africa. Tropical Doctor 2005;35:220-222.

7. McKenzie AG. Mortality associated with anaesthesia at Zimbabwean teaching hospitals. South African Medical Journal 1996;86:338-342.

8. Heywood AJ, Wilson IH, Sinclair JR. Perioperative mortality in Zambia. Annals of the Royal College of Surgeons of England 1989;71: 354-358.

9. Hansen D, Gausi SC, Merikebu M. Anaesthesia in Malawi: complications and deaths. Tropical Doctor 2000;30:146-149.

10. Gawande AA, et al. The incidence and nature of surgical adverse events in Colorado and Utah in 1992. Surgery 1999;126:66-75.

11. Kable AK, Gibberd RW, Spigelman AD. Adverse events in surgical patients in Australia. International Journal of Quality in Healthcare 2002;14:269-276.

12. Bickler SW, Sanno-Duanda B. Epidemiology of paediatric surgical admissions to a government referral hospital in the Gambia. Bulletin of the World Health Organization 2000;78:1330-1336.

13. McConkey SJ. Case series of acute abdominal surgery in rural Sierra Leone. World Journal of Surgery 2002;26:509-513.

14. Brennan TA, et al. Incidence of adverse events and negligence in hospitalized patients. Results of the Harvard Medical Practice Study I. New England Journal of Medicine 1991;324:370-376.

15. Wilson RM, et al. The quality in Australian healthcare study. Medical Journal of Australia 1995;163:458-471.

16. Leape LL, et al. The nature of adverse events in hospitalized patients—results of the Harvard Medical Practice Study II. New England Journal of Medicine 1991;324:377-384.

17. United Kingdom Department of Health. An organization with a memory. London; 2000.

18. Kable AK, Gibberd RW, Spigelman AD. Adverse events in surgical patients in Australia. International Journal of Quality in Healthcare 2002;14:269-276.

19. Ronsmans C, et al. Maternal mortality: who, where and why. Lancet 2006;368:1189-1200.

R Harsvardhan

Assistant Professor, Hospital Administration

Sanjay Gandhi Post Graduate Institute of Medical Sciences

Lucknow, Uttar Pradesh, India 